\title{
Clinical Progress Note: Point-of-Care Ultrasound for the Pediatric Hospitalist
}

\author{
Benjamin Kinnear, MD, MEd ${ }^{1 *}$, Matthew Kelleher, MD, MEd' ${ }^{1}$ Valeriy Chorny, MD²
}

${ }^{1}$ Department of Pediatrics, University of Cincinnati College of Medicine, Cincinnati, Ohio; ${ }^{2}$ Department of Pediatrics, SUNY Downstate and Kings County Hospital Center, Brooklyn, New York.

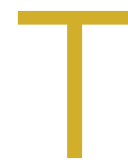

he recent designation of Pediatric Hospital Medicine (PHM) as a board-certified subspecialty has provided the opportunity to define which skills are core to hospitalist practice. One skill that is novel to the field and gaining traction is point-of-care ultrasonography (POCUS). POCUS differs from traditional ultrasonography in that it is performed at the bedside by the primary clinician and aims to answer a focused clinical question (eg, does this patient have a skin abscess?) rather than to provide a comprehensive evaluation of the anatomy and physiology. The proposed advantages of POCUS include real-time image interpretation, cost savings, procedural guidance to minimize complications, and reduction of ionizing radiation. Although specialties such as Critical Care (CC) and Emergency Medicine (EM) have integrated POCUS into their practice and training, best practices in PHM have not been defined. This Progress Note is a summary of recent evidence to update past reviews and set the stage for future PHM POCUS research and education.

\section{LITERATURE SEARCH STRATEGY AND TOPIC SELECTION}

We met with an academic librarian in March 2019 and performed a search of PubMed using Medical Subject Headings (MESH) terms associated with POCUS and Pediatrics. We limited our search to studies published within the past 5 years. The search was originally focused to the field of PHM before expanding to a broader search since very few studies were found that focused on Hospital Medicine or general pediatric ward populations. This initial search generated 274 publications. We then performed a supplemental literature search using references from studies found in our initial search, as well as further ad hoc searching in Embase and Google Scholar.

After our literature search, we reviewed the PHM core competencies and identified the common clinical diagnoses and core skills for which there is POCUS literature published in the past five years. These included acute abdominal pain, bronchiolitis, pneumonia, skin and soft-tissue infection, newborn care/delivery room management, bladder catheterization, fluid management, intravenous access, and lumbar puncture (LP).

*Corresponding Author: Benjamin Kinnear, MD, MEd; Email: kinneabn@ ucmail.uc.edu; Telephone: 513-541-4667

Published online first November 20, 2019

Received: May 3, 2019; Revised: August 30, 2019; Accepted: September 4, 2019

๑) 2020 Society of Hospital Medicine DOI 10.12788/jhm.3325
We chose to focus on one skill and two diagnoses that were generalizable to pediatric hospitalists across different settings and for which there was compelling evidence for POCUS use, such as pneumonia, skin abscess, and LP. We found few studies that included general pediatric ward patients, but we considered EM and CC studies to be relevant as several pediatric hospitalists practice in these clinical settings and with these patient populations.

\section{PNEUMONIA}

POCUS can be useful for diagnosing pneumonia by direct visualization of lung consolidation or by identification of various sonographic artifacts that suggest pathology. For example, "B-lines" are vertical artifacts that extend from the pleura and suggest interstitial fluid or pneumonia when they are present in abnormally high numbers or density. POCUS can also be used to diagnose parapneumonic effusions by scanning dependent areas of the lung (eg, the diaphragm in children sitting upright) and looking for anechoic or hypoechoic areas.

Three recent meta-analyses found favorable operating characteristics when using POCUS for the diagnosis of pneumonia in children, with summary sensitivities of $93 \%-94 \%$ and specificities of $92 \%-96 \% .^{1-3}$ However, these meta-analyses were limited by high heterogeneity caused by the inclusion of multiple different care settings and the use of variable reference standards and sonographic criteria for diagnosing pneumonia. POCUS is superior to chest radiography for evaluating parapneumonic pleural effusions, ${ }^{4}$ allowing for rapid identification of loculations, fibrin strands, and proteinaceous material, and for serial bedside evaluation of effusion size and characteristics.

Additional advantages of POCUS include avoidance of ionizing radiation and the potential for cost and time savings. Two studies demonstrated reductions in radiography use and improved cost, although they were not conducted on hospitalized patients. One randomized controlled trial (RCT) conducted in a pediatric emergency department (ED) demonstrated a $38.8 \%$ reduction in chest radiography use without increasing the ED length of stay (EDLOS), antibiotic use, or unscheduled follow-up visits. ${ }^{5} \mathrm{~A}$ retrospective matched cohort study conducted in another pediatric ED reported that, when compared with patients evaluated by chest radiography, those evaluated by POCUS had significantly shorter EDLOS (-60.9 min) and mean health systems savings ( $\$ 187$ per patient). ${ }^{6}$ We believe that POCUS has value in the evaluation and management of pneumonia and parapneumonic effusions, although further studies investigating patient outcomes and involving inpatient populations are required. 


\section{SKIN ABSCESS}

POCUS can augment the physical examination, helping to both avoid unnecessary incision and drainage (I+D) procedures and detect drainable fluid collections. Abscess is suggested when hypoechoic material without vascular flow is detected, and although other structures such as vessels, cysts, and lymph nodes can mimic skin abscesses, this is a relatively straightforward examination for clinicians to learn.

Two meta-analyses found that POCUS had high sensitivity for diagnosing skin abscesses in the ED.7,8 A pediatric subgroup analysis conducted in a study by Barbic et al. found a sensitivity and a specificity of $94 \%$ (95\% Cl: 88\%-98\%) and $83 \%$ (95\% Cl: 47\%-97\%), respectively. ${ }^{7}$ Subramaniam et al. included six studies (four pediatric) with 800 patients (653 $\leq 18$ years old) and found an overall pooled sensitivity of $97 \%$ (95\% Cl: $94 \%-$ $98 \%$ ) and a specificity of $83 \%$ (95\% Cl: $75 \%-88 \%) .{ }^{8}$ No subgroup analysis was performed, but the included pediatric studies reported sensitivities and specificities between 90\%-98\% and $68 \%-87 \%$, respectively.

Although POCUS performs better than physical examination for the diagnosis of drainable abscesses, evidence regarding patient outcomes is mixed. A retrospective review from four pediatric EDs found that integration of POCUS lowered treatment failure rates, defined as any incision and drainage (I+D) or surgical manipulation after discharge from the initial ED visit (4.4\% vs $15.6 \%$; $P<.005) .{ }^{9}$ A single-center retrospective cohort study found that POCUS reduced EDLOS by a median of 73 minutes (95\% Cl: 52-94 $\mathrm{min}$ ) when compared with radiologyperformed studies. ${ }^{10}$ The aforementioned study conducted by Barbic et al. found that, in pediatric studies, POCUS led to a change in management (eg, whether or not to attempt I+D) in $14 \%-27 \%$ of patients. ${ }^{7}$ However, a multicenter prospective observational cohort study involving seven pediatric EDs found that, despite changing the management in $22.9 \%$ of cases, POCUS was not associated with any statistically significant differences in treatment failure rates, EDLOS, discharge rates, use of sedation, or use of alternative imaging. ${ }^{11}$ These studies were limited by a lack of randomization or control group and emphasize the need for RCTs that measure patient outcomes. Future studies should investigate how POCUS can be used in inpatient settings both for initial diagnosis of drainable abscesses and for serial evaluation of evolving phlegmon or incompletely drained collections.

\section{LUMBAR PUNCTURE}

LP is commonly performed by pediatric hospitalists, although success can be influenced by numerous factors, including provider and staff expertise, patient anatomy, and body habitus. Requiring multiple attempts can increase patient discomfort and parental anxiety. Failure to obtain cerebrospinal fluid can delay diagnosis or leave providers in uncertain clinical situations that may commit patients to prolonged antibiotic courses. POCUS can be used to identify anatomic markers such as interspinous processes, anatomic midline, and depth of the ligamentum flavum. ${ }^{12}$ It can also be used to identify epidural hematomas after failed LPs to avoid additional unsuccessful attempts. ${ }^{13}$ POCUS guidance for LP has been described using both static (preprocedural marking) and dynamic (scanning during the procedure) techniques, although most of the studies use the static approach. The Society of Hospital Medicine POCUS Task Force has recently released a position statement recommending that POCUS should be used for site selection before performing LP in adult patients when providers are adequately trained. ${ }^{12}$ Although this position statement was for adult patients, recent evidence suggests that there is also benefit in Pediatrics.

Two recent meta-analyses have investigated POCUS use for pediatric LPs. 14,15 Olowoyeye et al. included four studies with a total of 277 patients and found that POCUS use was associated with a reduction in traumatic taps (risk ratio $[R R]=0.53$, 95\% Cl: 0.13-0.82) when compared with landmark approaches. ${ }^{14}$ However, there was no statistically significant reduction in LP failure, number of needle insertion attempts, or procedure length. A more recent meta-analysis performed a pediatric subgroup analysis of six studies including 452 patients and found a statistically significant reduction in traumatic taps (13.7\% vs $31.8 \%$, risk difference $=-21.3 \%, 95 \% \mathrm{Cl}:-38.2 \%$ to $-4.3 \%$ ) and number of needle insertion attempts (1.53 vs 2.07, mean difference $=-0.47,95 \% \mathrm{Cl}:-0.73$ to -0.21$).{ }^{15}$ The primary outcome of LP success trended toward favoring POCUS, but it was not statistically significant $(88.4 \%$ vs $74.0 \%, \mathrm{OR}=2.55$, 95\% Cl: 0.99-6.52). We believe that recent evidence suggests that there is benefit in using POCUS when hospitalists attempt pediatric LPs, particularly when physical landmarks are difficult to identify or after failed attempts. However, adequate training with simulation and supervised practice should be undertaken before integrating this into clinical practice.

\section{CONCLUSION}

Evidence accumulated in the past 5 years has built on previous work suggesting that POCUS has a role in the diagnosis of pneumonia and skin abscess and in the performance of LPs. However, gaps in the literature remain when applying POCUS in PHM. Only a few studies to date were conducted in non-CC inpatient settings, and although several pediatric hospitalists work in EDs or care for critically ill children, our largest population comprises general pediatric ward patients. Studies have also used ultrasonographers with variable POCUS training and clinical experience, which makes comparing or combining studies challenging since POCUS is dependent on provider skills. Studies involving PHM providers and inpatient populations are needed. Additional studies evaluating the process and outcome measures are also needed to understand whether the theoretical advantages are consistently realized in real-world PHM practice. Finally, PHM-specific curricula should be designed in collaboration with various PHM stakeholders and with specialties who already have robust POCUS training pathways. There is opportunity within PHM for multi-institutional research collaboration, identification of best practices, and development of PHM-specific training for fellowship and faculty development programs.

Disclosures: The authors have nothing to disclose. 


\section{References}

1. Orso D, Ban A, Guglielmo N. Lung ultrasound in diagnosing pneumonia in childhood: a systematic review and meta-analysis. J Ultrasound 2018;21(3):183-195. https://doi.org/10.1007/s40477-018-0306-5.

2. Najgrodzka P, Buda N, Zamojska A, Marciniewicz E, Lewandowicz-Uszynska A. Lung ultrasonography in the diagnosis of pneumonia in children-a meta-analysis and a review of pediatric lung imaging. Ultrasound Q. 2019; 35(2):157-163. https://doi.org/10.1097/RUQ.0000000000000411.

3. Xin H, Li J, Hu HY. Is lung ultrasound useful for diagnosing pneumonia in children? A meta-analysis and systematic review. Ultrasound Q. 2018;34(1):310. https://doi.org/10.1097/RUQ.0000000000000330.

4. Esposito S, Papa SS, Borzani l, et al. Performance of lung ultrasonography in children with community-acquired pneumonia. Ital J Pediatr. 2014;40(1):37. https://doi.org/10.1186/1824-7288-40-37.

5. Jones BP, Tay ET, Elikashvili I, et al. Feasibility and safety of substituting lung ultrasonography for chest radiography when diagnosing pneumonia in children: a randomized controlled trial. Chest. 2016;150(1):131-138. https://doi. org/10.1016/j.chest.2016.02.643.

6. Harel-Sterling M, Diallo M, Santhirakumaran S, Maxim T, Tessaro M. Emergency department resource use in pediatric pneumonia: point-of-care lung ultrasonography versus chest radiography. J Ultrasound Med. 2019;38(2):407414. https://doi.org/10.1002/jum.14703.

7. Barbic D, Chenkin J, Cho DD, Jelic T, Scheuermeyer FX. In patients presenting to the emergency department with skin and soft tissue infections what is the diagnostic accuracy of point-of-care ultrasonography for the diagnosis of abscess compared to the current standard of care? A systematic review and meta-analysis. BMJ Open. 2017;7(1):e013688. https://doi.org/10.1136/ bmjopen-2016-013688.

8. Subramaniam S, Bober J, Chao J, Zehtabchi S. Point-of-care ultrasound for diagnosis of abscess in skin and soft tissue infections. Acad Emerg
Med. 2016;23(11):1298-1306. https://doi.org/10.1111/acem.13049.

9. Gaspari RJ, Sanseverino A. Ultrasound-guided drainage for pediatric soft tissue abscesses decreases clinical failure rates compared to drainage without ultrasound: a retrospective study. J Ultrasound Med. 2018;37(1):131-136. https://doi.org/10.1002/jum.14318.

10. Lin MJ, Neuman M, Rempell R, Monuteaux M, Levy J. Point-of-care ultrasound is associated with decreased length of stay in children presenting to the emergency department with soft tissue infection. J Emerg Med. 2018;54(1):96-101. https://doi.org/10.1016/j.jemermed.2017.09.017.

11. Lam SHF, Sivitz A, Alade K, et al. Comparison of ultrasound guidance vs. clinical assessment alone for management of pediatric skin and soft tissue infections. J Emerg Med. 2018;55(5):693-701. https://doi.org/10.1016/j. jemermed.2018.07.010.

12. Soni NJ, Franco-Sadud R, Kobaidze K, et al. Recommendations on the use of ultrasound guidance for adult lumbar puncture: a position statement of the Society of Hospital Medicine [published online ahead of print June 10, 2019]. J Hosp Med. 2019;14:E1-E11. https://doi.org/10.12788/jhm.3197.

13. Kusulas MP, Eutsler EP, DePiero AD. Bedside ultrasound for the evaluation of epidural hematoma after infant lumbar puncture [published online ahead of print January 2, 2018]. Pediatr Emerg Care. 2018. https://doi.org/10.1097/ PEC.0000000000001383.

14. Olowoyeye A, Fadahunsi O, Okudo J, Opaneye O, Okwundu C. Ultrasound imaging versus palpation method for diagnostic lumbar puncture in neonates and infants: a systematic review and meta-analysis. BMJ Paediatr Open. 2019;3(1):e000412. https://doi.org/10.1136/bmjpo-2018-000412.

15. Gottlieb M, Holladay D, Peksa GD. Ultrasound-assisted lumbar punctures: a systematic review and meta-analysis. Acad Emerg Med. 2019;26(1):85-96. https://doi.org/10.1111/acem.13558. 